\title{
Using deceased-donor kidneys to initiate chains of living donor kidney paired donations
}

DOI:

$10.1145 / 3306618.3314276$

\section{Document Version}

Accepted author manuscript

Link to publication record in Manchester Research Explorer

\section{Citation for published version (APA):}

Cornelio, C., Nicolò, A., Furian, L., \& Rossi, F. (2019). Using deceased-donor kidneys to initiate chains of living donor kidney paired donations: Algorithms and experimentation. In AIES 2019 - Proceedings of the 2019

AAAl/ACM Conference on Al, Ethics, and Society (pp. 477-483). (AIES 2019 - Proceedings of the 2019 AAAl/ACM Conference on Al, Ethics, and Society). Association for Computing Machinery.

https://doi.org/10.1145/3306618.3314276

Published in:

AIES 2019 - Proceedings of the 2019 AAAI/ACM Conference on Al, Ethics, and Society

\section{Citing this paper}

Please note that where the full-text provided on Manchester Research Explorer is the Author Accepted Manuscript or Proof version this may differ from the final Published version. If citing, it is advised that you check and use the publisher's definitive version.

\section{General rights}

Copyright and moral rights for the publications made accessible in the Research Explorer are retained by the authors and/or other copyright owners and it is a condition of accessing publications that users recognise and abide by the legal requirements associated with these rights.

\section{Takedown policy}

If you believe that this document breaches copyright please refer to the University of Manchester's Takedown Procedures [http://man.ac.uk/04Y6Bo] or contact uml.scholarlycommunications@manchester.ac.uk providing relevant details, so we can investigate your claim.

\section{OPEN ACCESS}


Title page

Deceased donor-initiated Chains: first report of a successful deliberate case and its ethical implications

Lucrezia Furian1 M.D.

Cristina Cornelio2 Ph.D

Cristina Silvestre1 M.D., Ph.D.

Flavia Neri1 M.D.

Francesca Rossi3, Ph.D.

Paolo Rigotti1 M.D.

Emanuele Cozzi4,M.D., Ph.D.

Antonio Nicolò5 Ph. D.

1 Kidney and Pancreas Transplantation Unit - Department of Surgical, Oncological and Gastroenterological Sciences, University of Padova, Italy

2 IBM T.J. Watson Research Center, Yorktown Heights, New York, U.S.

3 IBM T.J. Watson Research Center Yorktown Heights, New York, U.S., and University of Padova, Italy

4Transplant Immunology Unit, Padua University Hospital, Italy

5 Department of Economics and Management, University of Padova, Italy and School of Social Sciences, University of Manchester, UK

Corresponding author:

Lucrezia Furian, MD

Kidney and Pancreas Transplantation Unit

Department of Surgical, Oncological and Gastroenterological Sciences

University of Padova

Via Giustiniani 2, 35128

Tel +390498211759 Fax +390498213152

Padua -ITALY

Email: Lucrezia.furian@unipd.it 
Authorship page

Authorship:

Lucrezia Furian: had the original idea, designed the study and wrote the paper; Cristina Cornelio: designed the study and analyzed data; Cristina Silvestre: participated in data collection; Flavia Neri: participated in data collection; Francesca Rossi: analyzed data; Paolo Rigotti: designed the study, performed the case and contributed to data interpretation; Emanuele Cozzi: data interpretation and review of the manuscript; Antonio Nicolò: designed the study and wrote the paper;

The Authors declare no conflicts of interest

Funding: Progetti Strategici di Ateneo -bando 2011, University of Padua

"KIDNEY -Incorporating patients' preferences in kidney transplant decision protocols" 
Abbreviations page

CDC, complement dependent cytotoxicity

CIK, chain initiating kidney

DBD, Donor after brain-dead

DEC-K, KPD started from DD kidney

DD, Deceased donor

DSA, Donor Specific Antibodies

ESRD, end-stage renal disease

HLA, human leucocyte antigen

KDPI, Kidney Donor Profile Index

KDRI, Kidney Donor Risk Index

KPD, Kidney Paired Donation

$\mathrm{KT}$, kidney transplant

LKDPI, Live Kidney Donor Profile Index

NITp, Nord Italia Transplant program

NT, no-transplanted recipients

PNI, national program for immunized patients

UT, unlikely transplantable recipients

WL, waiting list 


\section{Abstract}

Background: The utilization ofdeceased donor kidneys to initiate chains of living donor kidney paired donation (KPD) has been proposed, although the potential gain of this practice needs to be quantified and the ethical implications must be addressed before starting its application.

Methods: The gain of implementing deceased donor-initiated chains has been measured through a mathematical algorithm, using retrospective data on the pool of donor/recipient incompatible pairs at a single Center. Allocation rules of chain ending kidneys and characteristics/quality of the chain initiating kidney (CIK) are described.

Results: the quantification of benefit analysis showed that with a pool of 69 kidneys from deceased donors and 16 pairs enrolled in the KPD program, over a period of 3 years it is possible to transplant 8/16 recipients (50\%). Following the approval of the Bioethical Committee of the Veneto Region and the revision of the allocation policies by the Italian National Transplant Center, the first successful case has been performed. The waiting time of the recipient (male, 53 yo) after entering the program for the $\mathrm{CIK}$, with a kidney donor risk index (KDRI) equal to 0.61 and a kidney donor profile index (KDPI)of 3\%) was 4 days; his willing donor (female, 53 yo, showed a living kidney donor profile index (LKDPI) of 2, and donated 2 days later, and the chain ending recipient (male, 47 yo,) was on dialysis for 5 years.

Conclusions: This is the first report of a deliberate deceased donor-initiated chain, which has been successfully performed. This has been made possible thanks to an extensive phase of evaluation of the ethical issues and allocation policy impact. This paper includes a preliminary efficacy assessment and the development a dedicated algorithm. 
Introduction

Living donor renal transplantation is the most promising solution for closing the gap between organ demand and supply. Despite growing efforts to implement this option for patients with end-stage renal disease (ESRD), the percentage of living donations has been decreasing in the United States from $50.1 \%$ in 2000 to $38.2 \%$ in 2017 of the total number of kidney transplants (1).

Figures are different in the European countries, where the expansion of living transplantation programs is still underway. ABO-incompatible or KPD programs are not fully and uniformly developed: beside the Dutch and the UK programs, that have already successfully optimized these processes, other European countries such as Spain, France, Italy, Czech Republic, Austria, Belgium, Switzerland, Poland and Scandinavia have just started these programs or are striving to implement one.

In Italy, 2.221 kidney transplants have been undertaken in 2017 and among these 310 were from living donors, with an increase of $61 \%$ compared to 2012 and 1.7 fold compared to 2007 (2), and desensitization techniques for ABO incompatibility are now applied in the highestvolume centres. However, KPD programs have only been performed in few cases, mainly due to the low number of patients enrolled in such programs. Nevertheless, KPD seems to represent the best option for candidates with circulating human leucocyte antigen (HLA) antibodies directed against their willing donors.

Several options to expand KPD have been proposed in the last few years, with dissimilar applicability: 1. A proposal for a nationwide and international KPD registry and match run, using optimization algorithms to match pairs, including three-way matches; 2 . The inclusion of altruistic donors to initiate chains of KPD: 3. The combination of KPD with desensitization to relax the requirement for a negative crossmatch for highly sensitized patients; 4 . The inclusion of compatible pairs into small single-centre pools to achieve match rates that might even surpass those attainable in a nationwide list made up of only incompatible pairs; 5 . List exchange and non-directed donation, where a living incompatible donor provides a kidney to a candidate on the deceased donor (DD) list and in return the list exchange intended recipient receives priority on the DD waiting list $(\mathrm{WL})(3,4)$.

More recently, the utilization of DD kidneys to initiate living donor chains has been proposed $(5,6)$, hypothesizing that a pilot program would show a positive impact also on patients on the DD waiting list, improving the quality of kidneys allocated to waitlisted patients, independently from ethnicities and blood types. However, important issues when implementing such program may raise ethical and logistical concerns: 1. The parameters of prioritization in the WL for the CIK recipient, 2. The acceptable quality and risk characteristics of the donors of CIK, 3. The number of DD kidneys that might be used as CIKs, 4. The allocation strategies of the chain ending kidney, 5 . The estimation of the quality of the living donor kidney compared to that of a DD kidney, 6 . The risk for reneging of the living donors due to the non- 
simultaneity of the operations, 7 . The impact of cold ischemia time in the realization of the chains in large regions.

As previously stated by Wall et al (6), in order to be implemented, such an innovation needs to take into consideration all these challenges. Here we describe how the ethical and logistical concerns were addressed in order to perform the first case of DD-initiated chain.

Methods

Quantification of benefit

In order to measure the potential gain of the program and to establish the number of DD-CIK that could ideally be diverted from the standard waiting list, we used retrospective data on the pool of donor/recipient incompatible pairs at a single centre. We simulated the gain of implementing KPD transplants at a local level, starting KPD chains from a DD organ, continuing by means of consecutive donations among pairs of incompatible living donorrecipients, and ending with a transplant to a patient on the WL for a DD organ who does not have a willing donor.

Our sequential algorithm was previously described (7) and the details of the studied population are available as Supplemental Documents.

In our simulation, the recipient of the DD organ could be any of the ESRD patients $(n=16)$ with an incompatible living donor who were not transplanted during the period. Receiving a transplant, even from a DD, would certainly represent a gain for those patients who were not transplanted, but one may argue that this policy would subtract organs from the pool available for waitlisted candidates. It should be pointed out, however, that the organ providedby the living donor of the last pair of the chain will terminate to a DD waitlisted patient. Therefore, at least in theory, waitlisted patients without a potential living donor do not suffer from the introduction of this allocation procedure also because the expected graft survival of a living donor kidney is higher than that of a DD organ (8). Nevertheless, there has to be some equity warranted for the waitlisted candidates with a lower probability of finding a compatible organ. We identified two categories of waitlisted candidates who merit special consideration. These are represented by recipients unlikely to be transplanted for immunological reasons (UT recipients) and by blood-type 0 recipients.

UT recipients were defined based on the classification of the NITp, which considers UT recipients those patients on the WL for more than 5 years or on dialysis for more than 7 years due to immunological reasons (PRA $>80 \%$ ). During the period of study, 35 UT recipients were listed at our Centre and were considered available for running the algorithm. UT recipients deserve absolute protection against any detrimental effect of the procedure, as also a favourable treatment to increase their chance of receiving an organ, because they have already experienced a long waiting time; blood-type 0 recipients, who may experience longer waiting time than other waitlisted candidates due to the unbalanced distribution of blood-type 
0 donors, should not be at a disadvantage. We therefore added three constraints to our algorithm. First, DD organs that were directly allocated to UT recipients were excluded from the algorithm, and we only considered organs allocated to patients in the standard WL. Second, whenever there was more than one living donor chain with maximal length, we select the one, if any, that ended with an UT recipient. Third, if a blood-type 0 organ was used to start the donor chain, then the chain had to end with either an UT recipient or return a bloodtype 0 organ to the standard WL.

\section{Allocation policy and characteristics of CIK}

For a proper allocation of the CIK, the following immunological variables of donors and recipients were taken into account: $A B 0$ identity, HLA typing for both class I $(A, B, C)$ and class II (DP, DQ, DR) antigens; , unacceptable antigens were those with demonstrated single antigen beads assay (Luminex®) with MFI $>3000$.

Using DD kidneys for chain initiation by incorporating this program into the allocation algorithms would change patient selection. Indeed, even if the kidney at the end of the chain is returned to a patient on the DD donor waiting list, it would probably not be allocated to the patient who would have been at the top of the match list if this program had not been implemented. Taking due account of such a possible flaw, we defined for patients on the DEC-K program a DD allocation strategy that would not disadvantage specific patient categories with higher allocation priority. In the inter-regional NITp program, that covers an area of more than 19 million inhabitants, DD kidneys are allocated to a single WL with an overall number of about 2.800 patients. Recipients in the DEC-K program could be selected for a given organ only if such an allocation was not competing with high-priority national programs such as the urgency program (ESRD patients with lack of vascular access for dialysis), the PNI (national program for immunized patients), the kidney-pancreas program, or other inter-regional priorities (0-1 HLA mismatched or UT recipients).

The quality of the CIK was calculated with the KDRI and the KDPI calculator, and the score was expected to be comparable with the LKDPI of their willing living donor. Moreover, the DDs were defined as standard in terms of infectious or neoplastic risk.

The living donor kidney ending the chain was assigned according to the Italian allocation policies: in the absence of recipient in emergency list or PNI compatible patients, the graft was allocated with the NITK4 algorithm, which takes into account blood type, HLA matches and time on dialysis (9). 
In order to keep as short as possible the cold ischemia time of the $\mathrm{CIK}$, the pilot program was limited to a single Centre (Padua University Hospital) and its region of procurement (Veneto region). A CDC crossmatch was performed with a recipient current serum.

The ethical implications were clearly and extensively exposed to the Bioethical Committee of the Veneto Region (exempting further IRB approval) obtaining a favorable opinion in November 2017, and the program was defined to conform to the principle of benevolence, with high social and healthcare value. A specific informative and consent form for the recipients and their willing donors was elaborated with the collaboration of psychologists, bioethical and legal medicine experts. It was explained to the involved subjects by two physicians of the Transplant team (one surgeon and one nephrologist). When the CIK became available, before starting the surgical procedures, a confirmation of the consent was requested to both subjects, to avoid the risk of withdrawal of the living donor.

Results

Quantification of benefit

Results of the retrospective simulation are summarized in Table 1. Given a cohort of 16 incompatible pairs, and a pool of 69 standard DDs allocated to the Padua Transplant Centre, it turns out that by using 7 grafts from DDs to start a chain, it was theoretically possible to transplant $50 \%$ of the patients who would have not been otherwise able to receive a transplant in a time span of three years. This means that only $10 \%$ of the entire pool of standard grafts available was utilized to enter the program.

Moreover, in most of the cases (6/7) the chains ended to a UT recipient who therefore received a living donor kidney instead of a DD organ.

Report of the first case

The recipient was a 53 year old male, affected by ESRD due to IgA nephropathy who had received a first kidney transplant from a deceased donor in 2003 and had lost his graft function for chronic damage. He restarted hemodialysis in May 2017. His blood type was A negative, and he had received previous blood transfusions. His pre-transplant workup displayed no contraindications to a second kidney transplant. His 53 year old wife (blood type A positive) who intended to donate him a kidney and presented no clinical or psychological contraindications to donation, had CDC positive crossmatch, and the Luminex ${ }^{\circledR}$ single bead assay demonstrated DSA (anti-A2, MFI: 20.185; DQ06:03, MFI: 1.368). Moreover, HLA-A2 was a repeated mismatch with his previous transplant. The couple was enrolled in the cross over national program KPD in October 2017, and the patient was active in the WL for a DD since then. The couple was offered the option to enter the DEC-K program in February 2018, and completed the psychological and immunological work-up on March 9th 2018. 
Four days later, a deceased CIK was offered to the patient. The donor was a 28 year old white male, height $183 \mathrm{~cm}$, weight $94 \mathrm{~kg}$, DBD due to a head trauma, with no history of hypertension or diabetes, HCV negative, and serum creatinine of $0.57 \mathrm{mg} / \mathrm{dL}$ (KDRI 0.61 , KDPI 3\%), 2 HLA A mismatches, 2 HLA B, 1 HLA DR, 1DQ, 2 DP mismatches.

The kidney transplant was performed the next day with standard technique; cold ischemia time was 6 hours and the post-operative course was uneventful, with immediate recovery of renal function. The patient was discharged on post-operative day 9 with a serum creatinine of $0.9 \mathrm{mg} / \mathrm{dL}$.

Two days after her husband's transplant, the wife underwent laparoscopic left nephrectomy, without complications, and she was discharged on post-operative day 3.

The living donor kidney was allocated following the NITp algorithm to a WL recipient (male, 47 yo, group A, positive). The chain-ending patient was at his first transplant, on dialysis since April 2013, affected by Schoenlein Henoch purpura, height $165 \mathrm{~cm}$, weight $62 \mathrm{~kg}$. The LKDPI calculated for the pair was 2, being the donor non hypertensive, non-smoker, with eGFR 102 $\mathrm{ml} / \mathrm{min}$, BMI 20.5, 2 HLA-B mismatches and 1 DR mismatch.

The kidney transplant procedure was performed with a cold ischemia time of 1 hour and 45 minutes, renal function recovered promptly The CIK recipient did not display any complication and was discharged on post-operative day 10 , with a serum creatinine of $1.0 \mathrm{mg} / \mathrm{dL}$.

Discussion

The utilization of DD grafts as source of CIK to start chains has been proposed previously ( 5 , 6). The underlying ethical issues have been detailed and can be summarized as follows: 1 . The proper allocation strategy of a CIK from a DD and of the chain ending kidney, 2. The value of exchanging a living donor kidney for a DD kidney, 3. The risk of living donor withdrawal, 4 . The consent of the parties.

The precedent for using a DD kidney in kidney exchanges has been reported by Delmonico et al (10). Their experience, however, can be better described as a List Exchange procedure, since "following transplant of the kidney from the living donor to the highest ranking appropriate individual identified by the transplant center's list, the incompatible recipient for whom the donor kidney was originally intended receives the right of first refusal for the next ABO identical (crossmatch negative) deceased donor kidney available within the Region". Contrary to the present report, in the list exchange one will donate before his intended 
recipient receives an organ from a $\mathrm{DD}$, and the degree of uncertainty of the prioritization in the WL, mostly in case of highly sensitized patients as those involved in our program would become an ethical concern when asking a donor to anticipate his donation.

Nevertheless, the allocation strategy of the chain ending kidney was following the rules of their match run, and a similar approach has been used in our experience, according to the allocation strategy of the NITp (NITK4 algorithm).

More challenging, in our opinion, is the selection of the adequate CIK from a DD. This decision should take into account the expected graft survival and the risk of disease transmission of a DD kidney compared to a living donor kidney. Our protocol, therefore, considers the KDRI and the KPDI score of the DD kidney and compares them with the LKDPI (11) of the subsequent matches; it also excludes those donors with risk for transmissible disease. The predictive value of the LKDPI compared with KDPI of DD has been recently validated in a European cohort, and based on these findings, corresponding subgroups of LKDPI and KDPI showed comparable graft survivals (12). Nevertheless, each CIK and LD should be evaluated comprehensively, taking into account several clinical aspects which are not currently included in the indexes such as male/female and donor/recipient weight ratio, HLA mismatches, pre-emptive status of the recipients, specific comorbidities of the donors.

One further argument to address is the quantification of the diversion of kidneys from patients on the WL. In our simulation, we have observed that in the retrospective population of DD and incompatible pairs, only $10 \%$ of the standard DD kidneys ( 7 grafts) would have been diverted from the WL allowing to transplant $50 \%$ of the incompatible pairs. Furthermore, our approach would have returned to the donor pool 7 additional high quality grafts (from living donors), which would have been transplanted into UT or blood group 0 patients. The usefulness of the proposed program results undeniable, since it increases the overall number of kidneys available for transplantation and, consequently, the aggregate quality and quantity of life of ESRD patients.

One last important issue to address is the management of the consent process for all the involved subjects. The key points of an adequate information have to make clear to the prospective living donors and CIK recipients the level of prioritization that the program allows, the quality of the DD grafts considered and the respect of their freedom to withdrawal at any time of the program. It emerges of paramount prominence to avoid the occurrence of donor reneging, given that the simultaneity of more surgeries would be logistically very difficult to achieve within the DEC-K program. To minimize this risk, both the donor and the recipient of each pair undergo an extensive education by two physicians in a very standardized fashion (one surgeon and one nephrologist), then they undergo a specific psychological evaluation and finally they meet a "third party" committee, aimed to assess their degree of understanding of the program and their commitment to participate. 
However, it is noteworthy that, differently from in list exchanges, patients participating in a chain of donations receive a kidney before her/his donor donates an organ to another patient. Moreover, for each incompatible pair, who can start a chain of donations, it is possible to compute in advance which is the optimal chain to perform, making the logistics easier. Infact, the crossmatches can be performed as soon as the result of the algorithm run is available, given that the virtual crossmatch is expected negative. In this way, when a DD CIK is available, there is no need to repeat the crossmatch between the enrolled pairs, but only between the DD and the first recipient of the chain. The sera of the potential recipients are collected every three months by national policy, and the single antigen beads assay (Luminex®) will be repeated on each serum in a timely manner. Although the maximum flexibility can be applied within the protocol in terms of logistical organization, the most favorable option is to make the kidney travel: this does not seem to determine a problem in terms of cold ischemia time, depending evidently on the territory involved in the program. Our choice was to use a local pool of DD to minimize cold ischemia time (in the reported case CIT was 5 hours), whereas the transplants from LD can be organized similarly to what happens when the chain starts with an altruistic donor.

It has to be acknowledged that unspecified LKD to initiate exchange program pose several advantages compared to a program of DD initiating chains, as well as less (or at least different) ethical dilemmas, for many reasons: all patients receive a LD, WL recipients will always benefit, it logistically easier to plan. Nevertheless, there are some differences among Countries in the legislation, and for certainCountries, for example Germany, altruistic donation is prohibited by law. For other Countries, such as Italy, the obstacle of unspecified donation is not related to the law, but to the small number of altruistic donations, that are scattered events. The impulsion to explore further options to expand domino chains between incompatible pairs was, in our case, the scarcity of unspecified LKDs.

The present report represents, to the best of our knowledge, the first case of a chain of kidney transplants initiated by a DD kidney. Differently from previous experiences (12), this case was deliberate and programmed following a straightforward process aimed to address ethical and logistical issues. The extension of the program to a national level will confidently allow to optimize the allocation strategies and obtain longer chains, thus minimizing waiting times for highly immunized recipients with incompatible willing donors. 
Acknowledgments: Alessandro Nanni Costa M.D. and Massimo Cardillo M.D. for their contribution to the development of the project as Director of the National Transplant Center (CNT) and of the Nord Italia Transplant program, respectively. 
References

1. OPTN data as of January 12, 2017 (https://optn.transplant.hrsa.gov/data/view-datareports/national-data)

2. CNT (Centro Nazionale Trapianti) National Data, Italy. http://www.trapianti.salute.gov.it/imgs/C_17_pubblicazioni_2591_allegato.pdf

3. Roth $\mathrm{AE}$, Sonmez $\mathrm{T}$, Unver $\mathrm{MU}$, et al. Utilizing list exchange and non-directed donation through "chain" paired kidney donation. Am J Transplant 2006; 6:2694-2705

4. Veale JL, Capron AM, Nassiri $\mathrm{N}$ et al. Vouchers for future kidney transplants to overcome "chronological incompatibility" between living donors and recipients. Transplantation 2017; 101 (9): 2115-2119.

5. Melcher ML Roberts JP, Leichtman $A B$, et al. Utilization of deceased donor kidneys to initiate living donor chains. Am J Transplant. 2016 May;16(5):1367-70.

6. Wall AE, Veale JL, Melcher ML. Advanced donation programs and deceased donorinitiated chains- 2 innovations in kidney paired donation. Transplantation 2017; 101 (12): 2818-2824

7. Silvestre C, Nicolò A. Cornelio $C$ et al. Potential gain of utilizing kidneys from deceased donors to initiate "chain" kidney paired donations: quantification of benefit through a realworld retrospective analysis. Transplant Int 2017; 30 S2:82

8. Hart A, Smith JM, Skeans MA, et al. Kidney. Am J Transplant. 2016 Jan;16 Suppl 2:11-46

9. Poli $F$, Cardillo $M$, Scalamogna $M$. Clinical relevance of human leukocyte antigen antibodies in kidney transplantation from deceased donors: the North ItalyTransplant program approach. Hum Immunol. 2009 Aug;70(8):631-5.

10. Delmonico FL, Morrissey PE, Lipkowitz GS et al. Donor Kidney Exchanges. Am J Transplant 2004; 4:1628-1634

11.Massie AB, Leanza J, Fahmy LM et al. A risk index for living donor kidney transplantation Am J of Transplant 2016;16:2077-2084

12. Rehse G, Halleck F, Khadzhynov D et al, Validation of the living kidney donor profile index in a European cohort and comparison of long-term outcomes with US results. NDT 2018; 1-8

13. Flechner SM, Leeser $\mathrm{D}$, Pelletier $\mathrm{R}$ et al. The incorporation of an advanced donation program into kidney paired exchange: initial experience of the National Kidney Registry Am J Transplant 2015; 15:2712-2717

14. Pierobon ES, Sandrini S, De Fazio N, et al. Optimizing utilization of kidneys from deceased donors over 60 years: five-year outcomes after implementation of a combined clinical and histological allocation algorithm. Transpl Int. 2013 Aug;26(8):833-41. 



\begin{tabular}{|l|l|}
\hline Deceased donor kidneys used to start a chain & $7 / 69(10 \%)$ \\
\hline NT patients who received an organ & $8 / 16(50 \%)$ \\
\hline UT patients who received an organ & $6 / 35(17 \%)$ \\
\hline Living donor kidneys returned to the standard waiting-list. & 1 \\
\hline
\end{tabular}

Table 1: Results of the retrospective analysis 